\title{
A phase 2 multiple ascending dose study of the inhaled pan-JAK inhibitor nezulcitinib (TD-0903) in severe COVID-19
}

\begin{abstract}
Copyright $\odot$ The authors 2021.
This version is distributed under the terms of the Creative Commons Attribution Non-Commercial Licence 4.0. For commercial reproduction rights and permissions contact permissions@ersnet.org

Received: 5 March 2021 Accepted: 19 June 2021

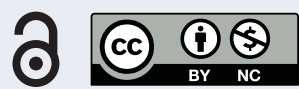

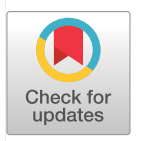

\section{To the Editor:}

Severe coronavirus disease 2019 (COVID-19) is characterised by pneumonia with excessive systemic inflammation, referred to as a "cytokine storm" [1-3]. Dexamethasone treatment decreases mortality in patients with COVID-19 receiving respiratory support and is standard of care for severe COVID-19 [4, 5]. However, pulmonary inflammation, which drives COVID-19 morbidity and mortality [3], can persist despite corticosteroid use [6, 7]. Janus kinase (JAK) inhibition blocks signalling by many cytokines in diverse cell types, offering broad immunomodulation [8]. The oral JAK-1/2 inhibitor baricitinib combined with the antiviral remdesivir shows clinical efficacy in patients with severe COVID-19 [9]. Direct delivery of JAK inhibition to the lung via inhalation could overcome corticosteroid-resistant pulmonary inflammation [10], offering the potential for improved responses while minimising risk of excessive systemic immunosuppression. The novel inhaled pan-JAK inhibitor nezulcitinib (TD-0903) was designed to target all JAK isoforms (JAK1, JAK2, JAK3, TYK2; - log inhibition constant 29.2) and optimise delivery to the lungs while limiting systemic exposure (R. Sana and co-workers; unpublished results: abstract submitted to ERS International Congress, 2021). We report results from the completed part 1 of a 2-part phase 2 trial (NCT04402866) in hospitalised patients with severe COVID-19.

The study was designed with separate data reporting for parts 1 and 2. Part 1 was a randomised, double-blind, placebo-controlled, multiple-ascending-dose trial conducted in the UK, Moldova and Ukraine. The study documents were approved by independent ethics committees at each site. The study was conducted in accordance with the principles of Good Clinical Practice and the Declaration of Helsinki. Patients provided informed consent. Hospitalised patients aged 18 to 80 years with PCR-confirmed symptomatic COVID-19 (symptoms for 3-14 days) who required supplemental oxygen to maintain saturation $>90 \%$ were eligible; patients receiving JAK inhibitors or anti-interleukin-6 therapy were excluded (full inclusion/exclusion criteria: https://www.clinicaltrials.gov/ct2/show/NCT04402866). Patients were sequentially enrolled in three ascending-dose cohorts ( $\mathrm{n}=6$ active and 2 placebo per cohort) and received once-daily nezulcitinib $1 \mathrm{mg}$ (day 1 loading dose $2 \mathrm{mg}$ ), $3 \mathrm{mg}$ (day 1 loading dose $6 \mathrm{mg}$ ), or $10 \mathrm{mg}$ (no loading dose) or matched placebo via inhalation (Aerogen Solo+Ultra nebuliser system, Galway, Ireland) for up to 7 days, with follow-up through day 28. Day 1 loading doses for nezulcitinib $1 \mathrm{mg}$ and $3 \mathrm{mg}$ were administered to rapidly achieve pseudo-steady state in the lung.

Peripheral blood arterial oxygen saturation $\left(\mathrm{S}_{\mathrm{aO}}\right)$ was collected via pulse oximetry, and fraction of inspired oxygen $\left(F_{\mathrm{IO}_{2}}\right)$, vital signs, adverse events, and clinical status using an 8-point ordinal scale (OS) [11] were recorded daily through day 7 and on days 14, 21 and 28 and/or at hospital discharge. Physical examination and blood collection were performed on days 1 and 7; patient care-related laboratory evaluations through day 28 were included. Safety was assessed from vital signs, laboratory results, and treatment-emergent adverse events (TEAEs; coded per Medical Dictionary for Regulatory Activities v23.1). Plasma pharmacokinetic parameters were evaluated on days 1 and 7. The key pharmacodynamic outcome was change from baseline $S_{\mathrm{aO}_{2}} / F_{\mathrm{IO}_{2}}$ ratio; other clinical outcomes were considered exploratory.

A sample size of six active- and two placebo-treated patients per cohort was deemed appropriate to assess nezulcitinib safety and tolerability during dose escalation. Safety, $S_{\mathrm{aO}_{2}} / F_{\mathrm{IO}_{2}}$ ratio, and efficacy data were summarised as descriptive statistics using SAS v9.4 (SAS Institute, Cary, NC, USA).

\section{Shareable abstract (@ERSpublications)}

The inhaled lung-selective pan-JAK inhibitor nezulcitinib appears generally well tolerated in hospitalised patients with severe \#COVID-19, with trends for improved oxygenation and clinical status, shortened hospitalisation, and fewer deaths versus placebo https://bit.ly/35Xs1Rf

Cite this article as: Singh D, Bogus M, Moskalenko V, et al. A phase 2 multiple ascending dose study of the inhaled pan-JAK inhibitor nezulcitinib (TD-0903) in severe COVID-19. Eur Respir J 2021; 58: 2100673 [DOI: 10.1183/13993003.00673-2021].
\end{abstract}


TABLE 1 Key baseline data and outcomes

Placebo $(n=6)$

\begin{tabular}{|c|c|c|}
\hline & Nezulcitinib & \\
\hline $1 \mathrm{mg}(\mathrm{n}=6)$ & $3 \mathrm{mg}(\mathrm{n}=7)^{\#}$ & $10 \mathrm{mg} \mathrm{(n=6)}$ \\
\hline
\end{tabular}

\section{Baseline characteristics}

\begin{tabular}{|c|c|c|c|c|}
\hline Sex, male & $3(50.0)$ & $5(83.3)$ & $4(57.1)$ & $5(83.3)$ \\
\hline Age, years & $54.2 \pm 17.0$ & $59.5 \pm 15.5$ & $62.0 \pm 3.3$ & $52.8 \pm 13.4$ \\
\hline Race, white & $5(83.3)$ & $6(100)$ & $7(100)$ & $6(100)$ \\
\hline \multicolumn{5}{|l|}{ Country of enrolment } \\
\hline Moldova & 3 & 6 & 6 & 3 \\
\hline Ukraine & 2 & 0 & 0 & 2 \\
\hline UK & 1 & 0 & 1 & 1 \\
\hline Body mass index, $\mathrm{kg} \cdot \mathrm{m}^{-2}$ & $27.2 \pm 3.6$ & $32.6 \pm 7.1$ & $31.8 \pm 2.7$ & $34.3 \pm 3.4$ \\
\hline \multicolumn{5}{|l|}{ Comorbidities } \\
\hline Hypertension & $4(66.7)$ & $6(100)$ & $4(57.1)$ & $3(50.0)$ \\
\hline Sleep apnoea & $1(16.7)$ & $4(66.7)$ & $3(42.9)$ & $3(50.0)$ \\
\hline Diabetes mellitus & $3(50.0)$ & $3(50.0)$ & $3(42.9)$ & $1(16.7)$ \\
\hline COVID-19 symptom duration, days, median (IQR) & $7.5(3.0)$ & $6.5(3.0)$ & $8.0(4.0)$ & $7.5(2.0)$ \\
\hline $\mathrm{S}_{\mathrm{aO}_{2}} / F_{\mathrm{IO}_{2}}$ ratio & $284.5 \pm 63.6$ & $295.0 \pm 28.2$ & $282.5 \pm 55.5$ & $270.0 \pm 61.5$ \\
\hline \multicolumn{5}{|l|}{ oncomitant medication } \\
\hline Dexamethasone & $5(83.3)$ & $6(100)$ & $7(100)$ & $5(83.3)$ \\
\hline Heparin group & $4(66.7)$ & $6(100)$ & $7(100)$ & $6(100)$ \\
\hline Remdesivir & $1(16.7)$ & 0 & $1(14.3)$ & $1(16.7)$ \\
\hline \multicolumn{5}{|l|}{ ummary of TEAEs ${ }^{+}$} \\
\hline All TEAEs & $6(100)$ & $5(83.3)$ & $2(28.6)$ & $5(83.3)$ \\
\hline Related to study treatment & $1(16.7)$ & $2(33.3)$ & 0 & $3(50.0)$ \\
\hline \multicolumn{5}{|l|}{ linical endpoints $\$$} \\
\hline Change in $\mathrm{S}_{\mathrm{aO}_{2}} / F_{\mathrm{IO}_{2}}$ from day 1 to 7 & $-49.5 \pm 65.3$ & $108.9 \pm 87.9$ & $106.4 \pm 87.8$ & $11.2 \pm 106.3$ \\
\hline Alive and respiratory failure-free on day 28 & $4(66.7)$ & $5(83.3)$ & $6(85.7)^{f}$ & $6(100)$ \\
\hline Time to hospital discharge, days $\# \#$ & $22.5 \pm 6.4$ & $18.8 \pm 6.8$ & $15.3 \pm 6.7$ & $15.2 \pm 4.4$ \\
\hline
\end{tabular}

Clinical status $\operatorname{OS}^{f, \boldsymbol{}, \boldsymbol{\Psi}}$

\begin{tabular}{|c|c|c|c|c|}
\hline \multicolumn{5}{|c|}{ Day 7} \\
\hline 1 & 0 & 0 & 0 & 0 \\
\hline 2 & 0 & 0 & 0 & 0 \\
\hline 3 & $1(16.7)$ & 0 & $1(16.7)$ & 0 \\
\hline 4 & 0 & $4(66.7)$ & $2(33.3)$ & $2(33.3)$ \\
\hline 5 & $2(33.3)$ & $2(33.3)$ & $3(50.0)$ & $4(66.7)$ \\
\hline 6 & 0 & 0 & 0 & 0 \\
\hline 7 & $3(50.0)$ & 0 & 0 & 0 \\
\hline 8 & 0 & 0 & 0 & 0 \\
\hline \multicolumn{5}{|c|}{ Day $28^{f}$} \\
\hline 1 & $3(50.0)$ & $5(83.3)$ & $5(83.3)$ & $5(83.3)$ \\
\hline 2 & 0 & 0 & 0 & $1(16.7)$ \\
\hline 3 & 0 & 0 & 0 & 0 \\
\hline 4 & $1(16.7)$ & 0 & $1(16.7)$ & 0 \\
\hline 5 & 0 & 0 & 0 & 0 \\
\hline 6 & 0 & 0 & 0 & 0 \\
\hline 7 & 0 & 0 & 0 & 0 \\
\hline 8 & $2(33.3)$ & $1(16.7)$ & 0 & 0 \\
\hline
\end{tabular}

Data are shown as mean \pm SD or $n(\%)$, unless otherwise specified. \#: one patient discontinued the study due to subsequent negative PCR test for severe acute respiratory syndrome coronavirus 2 (SARS-CoV-2) and was replaced but included in analyses as available data permitted. ": includes heparins, enoxaparin, bemiparin and nadroparin. ${ }^{+}$: including patients who received $\geqslant 1$ dose of study drug analysed as treated. ${ }^{\S}$ : including all randomised patients analysed as randomised (intent-to-treat population). ${ }^{f}$ : the patient who discontinued the study due to negative PCR test for SARS-CoV-2 was known to be alive but with unknown clinical status at day 28 . Thus, this patient was not counted as alive and respiratory failure-free and was not included in analyses of ordinal scale (OS) on days 7 and 28. \#\#: for patients who died or were still hospitalised on day 28 , a time to discharge of 28 days was assigned. ${ }^{~}{ }^{\top}$ : 8-point OS: 1 , not hospitalised, no limitations on activities; 2 , not hospitalised, but with limitation on activities and/or requiring home oxygen; 3 , hospitalised, not requiring supplemental oxygen and no longer requiring ongoing medical care (including hospitalisation for infection control); 4, hospitalised, not requiring supplemental oxygen but requiring ongoing medical care (whether related or not to coronavirus disease 2019 (COVID-19)); 5, hospitalised, requiring supplemental oxygen; 6, hospitalised, on noninvasive ventilation or high-flow oxygen devices; 7 , hospitalised, on invasive mechanical ventilation or extracorporeal membrane oxygenation; 8 , death. IQR: interquartile range; $S_{\mathrm{aO}_{2}} / F_{\mathrm{IO}_{2}}$ : ratio of oxygen saturation measured by pulse oximetry to fraction of inspired oxygen; TEAE: treatment-emergent adverse event. 
25 patients enrolled (UK, 3; Ukraine, 4; Moldova, 18) and were randomised to receive nezulcitinib $1 \mathrm{mg}$ $(\mathrm{n}=6), 3 \mathrm{mg}(\mathrm{n}=7), 10 \mathrm{mg}(\mathrm{n}=6)$, or placebo $(\mathrm{n}=6)$. One patient receiving nezulcitinib $3 \mathrm{mg}$ was discontinued due to a negative SARS-CoV-2 PCR screening test returned after randomisation and was replaced per protocol. Baseline data, concomitant medications, TEAEs and clinical outcomes are summarised in table 1. Mean body mass index and proportion of men were lower in patients receiving placebo versus nezulcitinib, and mean age was lower in patients receiving placebo or nezulcitinib $10 \mathrm{mg}$ versus nezulcitinib $1 \mathrm{mg}$ or $3 \mathrm{mg}$. Almost all patients (92\%) received dexamethasone; three (12\%) received remdesivir. The majority of TEAEs were mild to moderate and resolved by end of study, with no apparent dose relationship. Serious TEAEs occurred in five patients through day 28, including COVID-19 progression in one placebo-treated patient; acute respiratory distress syndrome (ARDS) and fatal multiple organ dysfunction syndrome (MODS) in one placebo-treated patient; ARDS and fatal cardiac arrest in one placebo-treated patient; acute respiratory failure, ventricular fibrillation, and fatal MODS in one nezulcitinib 1 mg-treated patient; and ischaemic stroke in one nezulcitinib 3 mg-treated patient. No serious TEAEs were considered related to study treatment by the investigator. One patient receiving nezulcitinib $10 \mathrm{mg}$ discontinued treatment on day 4 due to elevated alanine aminotransferase that resolved without consequence. No other changes in vital signs or laboratory safety measures, including creatinine and haematologic parameters, were attributed to treatment. On day 7, mean systemic levels of inflammatory biomarker high-sensitivity C-reactive protein (hSCRP) and lung injury marker soluble receptor for advanced glycation end products (sRAGE) were lower versus baseline in patients treated with nezulcitinib $3 \mathrm{mg}$ and $10 \mathrm{mg}$ (range: hsCRP, 52\%-75\% reduction; sRAGE, 54\%-83\% reduction) versus placebo (41\% increase and $37 \%$ reduction, respectively). The mean steady state maximal plasma concentration of nezulcitinib at the highest dose was $19.0 \mathrm{ng} \cdot \mathrm{mL}^{-1}$, well below levels predicted to produce systemic JAK inhibition.

At baseline, all patients received supplemental oxygen via nasal prongs or mask (OS 5). During the 7-day treatment period, three (50\%) placebo-treated patients progressed to invasive mechanical ventilation (OS 7), but no nezulcitinib-treated patient declined in clinical status. After day 7, one patient treated with nezulcitinib $1 \mathrm{mg}$ and two placebo-treated patients died (OS 8; nezulcitinib 1 mg-treated patient on day 23 and placebo-treated patients on days 11 and 14), whereas clinical status remained stable or improved through day 28 in all patients treated with nezulcitinib $3 \mathrm{mg}$ or $10 \mathrm{mg} . \mathrm{S}_{\mathrm{aO}_{2}} / F_{\mathrm{IO}_{2}}$ ratio improved from baseline to day 7, proportion of patients alive and respiratory failure-free at day 28 was higher and mean time to hospital discharge was shorter in patients treated with nezulcitinib versus placebo (table 1).

This is the first clinical trial of an inhaled JAK inhibitor for COVID-19 treatment. Once-daily inhaled nezulcitinib for 7 days was generally well tolerated in patients with severe COVID-19. There were trends towards improvement in $S_{\mathrm{aO}_{2}} / \mathrm{F}_{\mathrm{IO}_{2}}$ ratio, respiratory failure-free survival at day 28, and mean time to hospital discharge in patients treated with nezulcitinib versus placebo. Overall mortality was $33 \%$ in placebo-treated patients versus $5 \%$ in nezulcitinib-treated patients. The small size of this early-phase clinical trial and consequent baseline differences in clinical characteristics limited evaluation of efficacy through between-group comparisons and formal control for potential confounders. Nevertheless, the low mortality and pattern of earlier clinical recovery with nezulcitinib versus placebo treatment suggests promise for targeting cytokine-driven pulmonary inflammation in patients with severe COVID-19 through pan-JAK inhibition. Notably, JAK inhibition may have additive anti-inflammatory effects in combination with corticosteroid treatment $[10,12]$, which almost all patients in the study received, and, thus, inhaled nezulcitinib may complement dexamethasone for treatment of patients with COVID-19.

Based on all evidence, inhaled nezulcitinib $3 \mathrm{mg}$ was advanced for further evaluation in part 2, a larger $(\mathrm{n} \approx 200)$ double-blind, placebo-controlled parallel-group phase 2 study in hospitalised COVID-19 patients requiring supplemental oxygen (OS 5-6) (NCT04402866).

Dave Singh $^{1}$, Maxim Bogus ${ }^{2}$, Valentyn Moskalenko ${ }^{3,4,5}$, Robert Lord $\oplus^{6}$, Edmund J. Moran ${ }^{7}$, Glenn D. Crater ${ }^{7}$, David L. Bourdet ${ }^{7}$, Nathan D. Pfeifer ${ }^{7}$, Jacky Woo ${ }^{7}$, Elad Kaufman ${ }^{7}$, David A. Lombardi ${ }^{7}$, Emily Y. Weng ${ }^{7}$, Tuan Nguyen ${ }^{7}$, Ashley Woodcock ${ }^{6}$, Brett Haumann ${ }^{8}$ and Rajeev Saggar ${ }^{7}$

${ }^{1}$ Medicines Evaluation Unit, University of Manchester, Manchester University NHS Foundations Trust, Manchester, UK. ${ }^{2}$ Arensia Exploratory Medicine SRL, Timofei Moșneaga Republican Clinical Hospital, Chișinău, Moldova. ${ }^{3}$ Arensia Exploratory Medicine, LLC, Kyiv City Clinical Hospital \#12, Kyiv, Ukraine. ${ }^{4}$ Oleksandrivska Kyiv City Clinical Hospital, Kyiv, Ukraine. ${ }^{5}$ Brovary Multidisciplinary Clinical Hospital, Brovary, Ukraine. ${ }^{6}$ University of Manchester, Manchester University NHS Foundations Trust, Manchester, UK. ${ }^{7}$ Theravance Biopharma US, Inc., South San Francisco, CA, USA. ${ }^{8}$ Theravance Biopharma UK Limited, London, UK. 
Acknowledgements: The authors and Theravance Biopharma, Inc., thank the patients and their families for their participation and Kyla Kennedy for clinical operations. Dave Singh, Robert Lord and Ashley Woodcock are supported by the National Institute for Health Research (NIHR) Manchester Biomedical Research Centre (BRC).

This study was registered at Clinicaltrials.gov with identifier NCT04402866. Theravance Biopharma (and its affiliates) will not be sharing individual de-identified participant data or other relevant study documents.

Author contributions: D. Singh, E.J. Moran, R. Lord, G.D. Crater, A. Woodcock, B. Haumann and R. Saggar contributed to study design and interpretation. D. Singh, M. Bogus, V. Moskalenko and R. Lord enrolled patients, collected data and contributed to interpretation. D.L. Bourdet and N.D. Pfeifer performed pharmacokinetic analyses and contributed to data interpretation. J. Woo and E. Kaufman contributed to data interpretation. D.A. Lombardi, E.Y. Weng and T. Nguyen performed statistical analyses and contributed to data interpretation. All authors reviewed the manuscript critically for intellectual content and approved the final version for submission.

Conflict of interest: D. Singh reports personal fees from Theravance Biopharma during the conduct of the study; and personal fees from AstraZeneca, Boehringer Ingelheim, Chiesi, Cipla, Genentech, GlaxoSmithKline, Glenmark, Menarini, Mundipharma, Novartis, Peptinnovate, Pfizer, Pulmatrix, Theravance Biopharma and Verona outside this work. M. Bogus is an employee of Arensia Exploratory Medicine SRL. V. Moskalenko is an employee of Arensia Exploratory Medicine, LLC. R. Lord reports an independent grant from Vertex Pharmaceuticals for an investigator-initiated study on gastro-oesophageal reflux, honoraria from the Manchester Adult CF Centre for speaking at a conference and travel awards from the European CF Society and British Thoracic Society. E.J. Moran is an employee of Theravance Biopharma US, Inc., and shareholder of Theravance Biopharma, Inc. G.D. Crater is a former employee of Theravance Biopharma US, Inc., and owns shares in Theravance Biopharma, Inc. D.L. Bourdet is an employee of Theravance Biopharma US, Inc., and shareholder of Theravance Biopharma, Inc. N.D. Pfeifer is an employee of Theravance Biopharma US, Inc., and shareholder of Theravance Biopharma, Inc. J. Woo is an employee of Theravance Biopharma US, Inc., and shareholder of Theravance Biopharma, Inc. E. Kaufman is an employee of Theravance Biopharma US, Inc., and shareholder of Theravance Biopharma, Inc. D.A. Lombardi is an employee of Theravance Biopharma US, Inc., and shareholder of Theravance Biopharma, Inc. E.Y. Weng is a former employee of Theravance Biopharma US, Inc., and owns shares in Theravance Biopharma, Inc. T. Nguyen is an employee of Theravance Biopharma US, Inc., and shareholder of Theravance Biopharma, Inc. A. Woodcock reports fees from Theravance Biopharma, Inc., for consulting on the study design. B. Haumann is a former employee of Theravance Biopharma UK Limited and shareholder of Theravance Biopharma, Inc. R. Saggar is an employee of Theravance Biopharma US, Inc., and shareholder of Theravance Biopharma, Inc.

Support statement: The study was funded by Theravance Biopharma Ireland Limited. Medical writing and editorial support were provided by Judith M. Phillips, of AlphaBioCom, LLC, and funded by Theravance Biopharma US, Inc. Funding information for this article has been deposited with the Crossref Funder Registry.

\section{References}

1 McElvaney OJ, McEvoy NL, McElvaney OF, et al. Characterization of the inflammatory response to severe COVID-19 illness. Am J Respir Crit Care Med 2020; 202: 812-821.

2 Sims JT, Krishnan V, Chang CY, et al. Characterization of the cytokine storm reflects hyperinflammatory endothelial dysfunction in COVID-19. J Allergy Clin Immunol 2021; 147: 107-111.

3 Wiersinga WJ, Rhodes A, Cheng AC, et al. Pathophysiology, transmission, diagnosis, and treatment of coronavirus disease 2019 (COVID-19): a review. JAMA 2020; 324: 782-793.

4 World Health Organization. Corticosteroids for COVID-19: Living guidance - 2 September 2020. www.who.int/ publications/i/item/WHO-2019-nCoV-Corticosteroids-2020.1 Date last accessed: 1 March 2021.

5 Horby P, Lim WS, Emberson JR, et al. Dexamethasone in hospitalized patients with Covid-19. N Engl J Med 2021; 384: 693-704.

6 Karki R, Sharma BR, Tuladhar S, et al. Synergism of TNF-alpha and IFN-gamma triggers inflammatory cell death, tissue damage, and mortality in SARS-CoV-2 infection and cytokine shock syndromes. Cell 2020; 184: 149-168.

7 Tliba O, Damera G, Banerjee A, et al. Cytokines induce an early steroid resistance in airway smooth muscle cells: novel role of interferon regulatory factor-1. Am J Respir Cell Mol Biol 2008; 38: 463-472.

8 Banerjee S, Biehl A, Gadina M, et al. JAK-STAT signaling as a target for inflammatory and autoimmune diseases: current and future prospects. Drugs 2017; 77: 521-546.

9 Kalil AC, Patterson TF, Mehta AK, et al. Baricitinib plus remdesivir for hospitalized adults with Covid-19. N Engl J Med 2020; 384: 795-807.

10 Southworth T, Plumb J, Gupta V, et al. Anti-inflammatory potential of PI3Kdelta and JAK inhibitors in asthma patients. Respir Res 2016; 17: 124. 
11 Beigel JH, Tomashek KM, Dodd LE, et al. Remdesivir for the treatment of Covid-19 - Final report. N Engl J Med 2020; 383: 1813-1826.

12 Rodriguez-Garcia JL, Sanchez-Nievas G, Arevalo-Serrano J, et al. Baricitinib improves respiratory function in patients treated with corticosteroids for SARS-CoV-2 pneumonia: an observational cohort study. Rheumatology (Oxford) 2021; 60: 399-407. 